\title{
The NanoWorkstation: Complementing FIB-SEM Tools with Micromanipulators
}

\author{
Andrew J. Smith ${ }^{1}$, Andreas Rummel ${ }^{1}$, Matthias Kemmler ${ }^{1}$, Klaus Schock ${ }^{1}$ and Stephan Kleindiek ${ }^{1}$ \\ ${ }^{1 .}$ Kleindiek Nanotechnik, Reutlingen, Germany
}

FIB-SEM microscopes are the go-to tools for a wide range of standard tasks in a multitude of scientific research as well as R\&D environments. These tasks include TEM sample prepration [1], a number of 3D imaging techniques (block-face imaging, 3D EDX, 3D EBSD, etc.), as well as in situ (electrical, mechanical) characterization of materials [2], among others. A number of in situ methods make use of specialized micromanipulators and plug-in tools in addition to the range of detectors available to researchers.

Piezo-driven micro- and nanopositioning systems are a perfect addition to FIB-SEM microscopes as they provide additional degrees of freedom for positioning the samples themselves, or for using the above mentioned specialized characterization tools. The use of piezo-crystals for generating motion provides a high degree of accuracy. Making the micromanipulator as small as possible yields stability (i.e. low drift, minimal vibration susceptibility) [3].

Applications for micromanipulators include nanoprobing experiments for electrical characterization [4], force measurements using force sensors mounted to the micromanipulators for micro- and nanomechanical studies, and a multitude of 'pick \& place' tasks where an experiment demands a microor nano-sized object be placed in a precise position for further investigation. TEM sample liftout (mentioned above) is the most common of the latter applications. At the same time, many other situations occur where precisely positioning a sample on a specifically structured substrate is necessary.

One of the experiments performed in the course of this work involved indenting a MWCNT using a micromanipulator equipped with a force sensor. Performing this type of experiment inside a FIB-SEM tool has various advantages: The force sensor's tip can be re-shaped or cleaned in situ (if necessary), individual CNTs can be cut and/or mounted to substrates or sensors, Having multiple viewing angles can be very helpful when mechanically interacting with the sample in order to determine the sample's and tool's spatial relationships.

The micromanipulator was mounted to the microscope stage's tilt axis yielding independent sample motion in the $\mathrm{X}, \mathrm{Y}$, and $\mathrm{R}$ directions while the micromanipulator's orientation regarding stage tilt was always the same - i.e. the micromanipulator's coordinate system remained synchronized with the sample's coordinate system. This is useful as it eases the process of addressing various ROIs (regiouns of interest) on the sample while the force sensor remains in the center of the microscope's field of view.

Figure 1 shows an overview image of the investigated MWCNTs that were randomly distributed on a Si wafer. A single MWCNT was located by moving the sample using the microscope's stage until a single MWCNT was centered in the field of view. Subsequently, the force sensor was centered in the field of view and lowered to be close to the sample surface (Figure 2). After initiating force recording, the force sensor's tip was brought into contact with the MWCNT and pressure was applied until the sample ruptured. At this point the recorded force was $8.1 \mu \mathrm{N}$. Figure 3 shows the recorded force vs. time plot during the experiment. 
With the presence of micromanipulators in the FIB-SEM, other modes of mechanical tests are also possible, including tensile and compression testing. Also, electrical characterizations can be performed such as four-point probing experiments for determining a sample's resistivity.

\section{References:}

[1] Burkhardt, C., et al, ESREF (2002).

[2] J Rösler, D Mukherji, K Schock, S Kleindiek, Nanotechnology 18 (2007), p. 125303

[3] S Kleindiek, US Patent US5568004 A (1996)

[4] Y Lin, Q Li, A Armstrong, and GT Wang, Solid State Communications 149 (2009), p. 1608

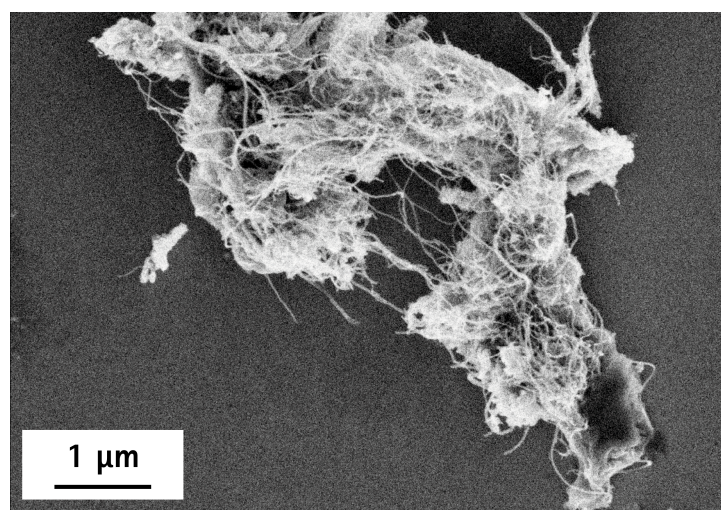

Figure 1. Overview image of MWCNT sample

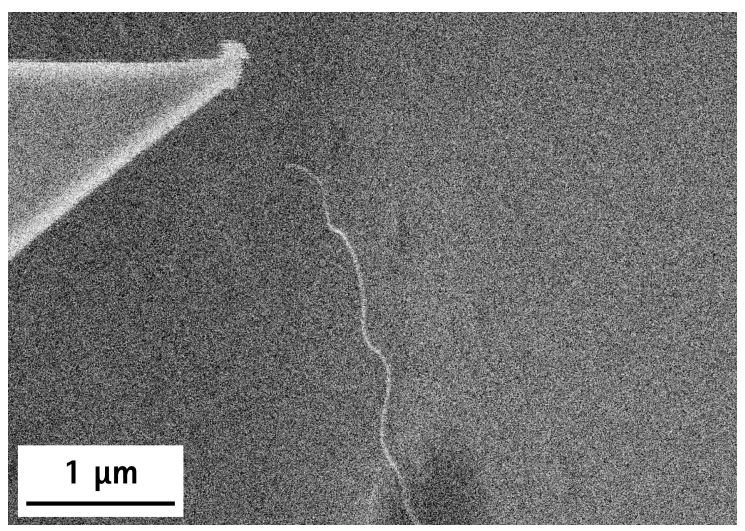

Figure 2. The force sensor in close vicinity to a single MWCNT

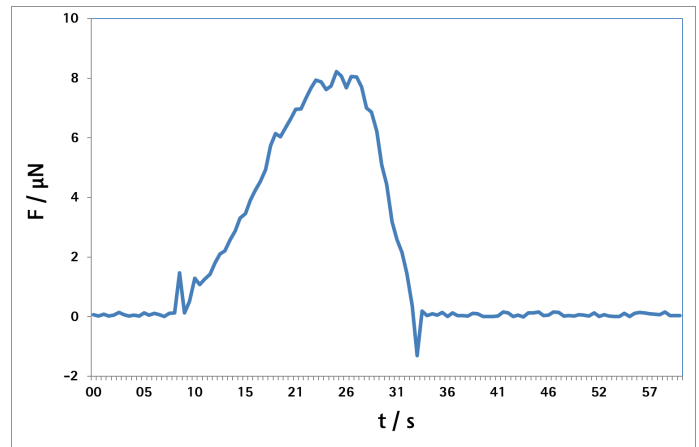

Figure 3. The force over time data recorded during the measurement 